CRYSTALLOGRAPHIC COMMUNICATIONS

ISSN 2056-9890

Received 10 December 2015

Accepted 26 January 2016

Edited by S. Parkin, University of Kentucky, USA

Keywords: crystal structure; indol-2-one; pyrazole; indole; Schiff base; $\mathrm{N}-\mathrm{H} \ldots \mathrm{O}$ and $\mathrm{O}-$ $\mathrm{H}$. . O hydrogen bonds; $\mathrm{C}-\mathrm{H} \cdots \pi$ interactions.

CCDC reference: 1450044

Supporting information: this article has supporting information at journals.iucr.org/e

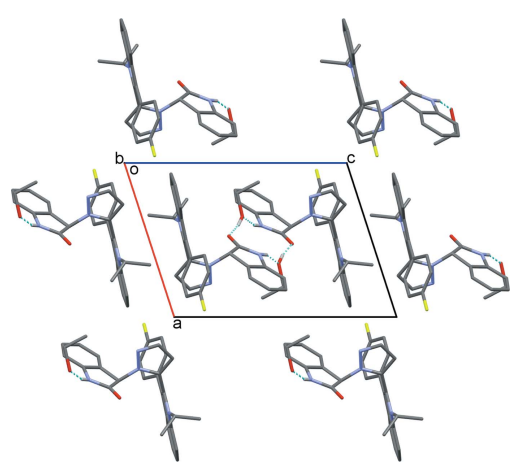

OPEN $\odot$ ACCESS

\section{Crystal structure of 3-\{5-[3-(4-fluorophenyl)-1- isopropyl-1H-indol-2-yl]-1H-pyrazol-1-yl\}indolin-2- one ethanol monosolvate}

\author{
Md. Lutfor Rahman, ${ }^{a *}$ Ajaykumar D. Kulkarni, ${ }^{b}$ Mashitah Mohd. Yusoff, ${ }^{a}$ \\ Huey Chong Kwong ${ }^{c}$ and Ching Kheng Quah ${ }^{d}$
}

aUniversity Malaysia Pahang, Faculty of Industrial Sciences and Technology, 26300 Gambang, Kuantan, Pahang, Malaysia, 'bepartment of Chemistry, KLS's Gogte Institute of Technology, Jnana Ganga, Udyambag, Belagavi-590008 Karnataka, India, ${ }^{\mathbf{C}}$ School of Chemical Sciences, Universiti Sains Malaysia, 11800 USM, Penang, Malaysia, and ${ }^{\mathbf{d} X-\text { ray }}$ Crystallography Unit, School of Physics, Universiti Sains Malaysia, 11800 USM, Penang, Malaysia. *Correspondence e-mail: lutfor73@gmail.com

The title indolin-2-one compound, $\mathrm{C}_{28} \mathrm{H}_{23} \mathrm{FN}_{4} \mathrm{O} \cdot \mathrm{C}_{2} \mathrm{H}_{6} \mathrm{O}$, crystallizes as a 1:1 ethanol solvate. The ethanol molecule is disordered over two positions with refined site occupancies of 0.560 (14) and 0.440 (14). The pyrazole ring makes dihedral angles of $84.16(10)$ and $85.33(9)^{\circ}$ with the indolin-2-one and indole rings, respectively, whereas the dihedral angle between indolin-2-one and indole rings is $57.30(7)^{\circ}$. In the crystal, the components are linked by $\mathrm{N}-\mathrm{H} \cdots \mathrm{O}$ and $\mathrm{O}-\mathrm{H} \cdots \mathrm{O}$ hydrogen bonds, forming an inversion molecule-solvate 2:2 dimer with $R_{4}^{4}(12)$ ring motifs. The crystal structure is consolidated by $\pi-\pi$ interaction between pairs of inversion-related indolin-2-one rings [interplanar spacing = 3.599 (2) ̊].

\section{Chemical context}

Heterocyclic compounds containing the pyrazolone nucleus, indole, and its derivatives play an important role in biological activities. The synthesis and biological activity of some new indole derivatives containing a pyrazole moiety have been reported (Raju et al., 2013). Pyrazole and its analogues have been found to exhibit industrial and biologically active applications (el-Kashef et al., 2000; Taha et al., 2001; Brzozowski \& Saczewski,, 2002). Consequently, synthesis of indole derivatives has been a major topic in organic and medicinal chemistry over the past few decades. Nitrogen-containing heterocycles are universal systems in nature and are consequently considered as privileged structures in drug discovery (Raju et al., 2013). A literature survey shows that some pyrazoles plays an essential role in biologically active compounds and also in medicinal chemistry (Penning et al., 2006), exhibiting phenomena such as antibacterial (Pevarello et al., 2006), antifungal, antiviral (Meghashyam et al., 2011), anti-oxidant (Singarave \& Sarkkarai, 2011), anti-inflammatory (Mana et al., 2010), and anticancer (Pathak et al., 2010) effects etc. Certain indole derivatives have also been reported to exhibit widespectrum activities such as antiparkinsonian and anticonvulsant effects (Siddiqui et al., 2008; Archana et al., 2002). In addition, pyrazoles have played a crucial role in the development of theory in heterocyclic chemistry, and are also used extensively as useful synthons in organic synthesis. Isatin, an endogenous indole and its derivatives have been shown to exhibit a wide range of biological activities (Daisley \& Shah, 
1984; Pandeya et al., 1999). In addition, the biological significance of fluvastatin, an indole derivative, is well established (Repič et al., 2001). As part of our studies in this area, we now present a pyrazole as a central unit linked with 3-[3-(4fluorophenyl)-1-isopropylindolin-2-yl]acrylaldehyde and 3-hydrazonoindolin-2-one, synthesized according to a procedure reported in the literature (Elkanzi, 2013).

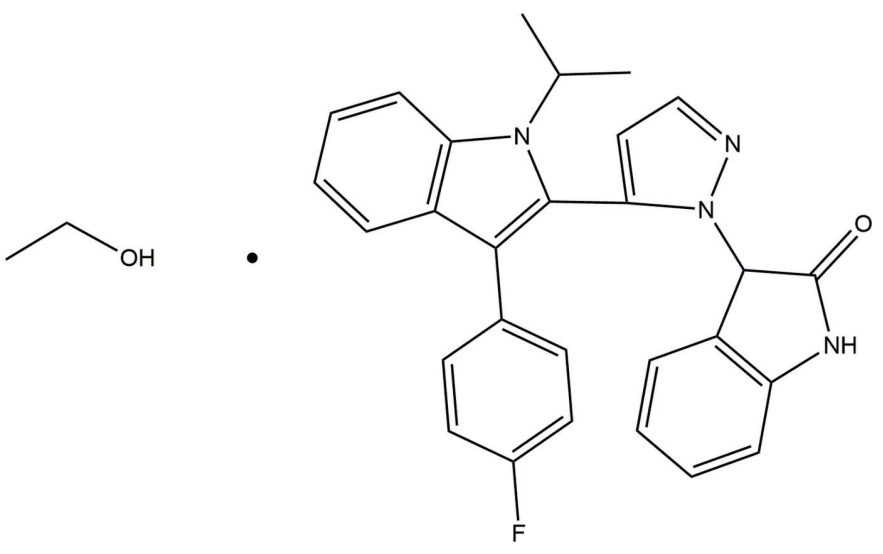

\section{Structural commentary}

The asymmetric unit of the title compound (Fig. 1) comprises of a 3-\{5-[3-(4-fluorophenyl)-1-isopropyl-1 $H$-indol-2-yl]- $1 H$ pyrazol-1-yl\}indolin-2-one and an ethanol solvent molecule. The pyrrolidin-2-one ring has an essentially planar conformation, with maximum deviation from the mean plane of the ring of 0.04 (2) $\AA$ at $\mathrm{C} 25$. The pyrazole ring is almost planar

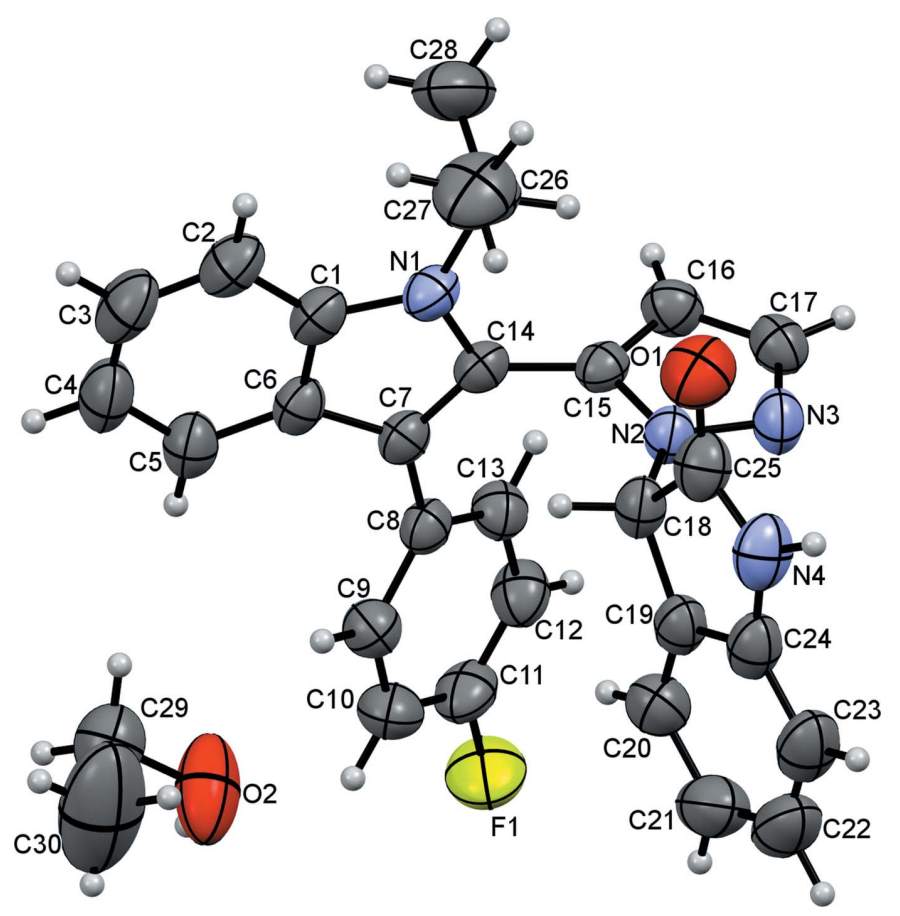

Figure 1

The molecular structure of the title compound. Displacement ellipsoids are drawn at the $30 \%$ probability level. Only the major component of the disordered ethanol solvent molecule is shown.
Table 1

Hydrogen-bond geometry $\left(\AA{ }^{\circ}\right)$.

\begin{tabular}{lllll}
\hline$D-\mathrm{H} \cdots A$ & $D-\mathrm{H}$ & $\mathrm{H} \cdots A$ & $D \cdots A$ & $D-\mathrm{H} \cdots A$ \\
\hline $\mathrm{N} 4-\mathrm{H} 1 N 1 \cdots \mathrm{O} 2^{\mathrm{i}}$ & $0.85(2)$ & $1.92(3)$ & $2.750(19)$ & $165(2)$ \\
$\mathrm{O} 2-\mathrm{H} 1 O 2 \cdots{ }^{\mathrm{ii}}$ & $0.98(9)$ & $1.67(9)$ & $2.650(2)$ & $172(11)$ \\
\hline
\end{tabular}

Symmetry codes: (i) $-x+1,-y+1,-z+1$; (ii) $x, y-1, z$

[maximum deviation of \pm 0.006 (2) $\AA$ for atoms N2 and C15], as are the fluorophenyl [maximum deviation of \pm 0.011 (2) $\AA$ for atoms $\mathrm{C} 10$ and $\mathrm{C} 13$ ] and indole [maximum deviation of \pm 0.0019 (2) $\AA$ for atom C14] rings. The connecting pyrazole ring is almost normal to both indol-2-one and indole rings with dihedral angles of $84.16(10)^{\circ}$ and $85.33(9)^{\circ}$, respectively, while the indole and fluorophenyl rings are tilted toward one another by $40.74(8)^{\circ}$. The bond lengths and angles in the fluorophenyl-indole moiety of the title molecule are comparable to those of previously reported compounds (Kulkarni et al., 2015a,b).

\section{Supramolecular features}

In the crystal, the main molecules and ethanol solvate molecules are linked via pairs of $\mathrm{N} 4-\mathrm{H} 1 N 1 \cdots \mathrm{O} 2$ and $\mathrm{O} 2-$ $\mathrm{H} 1 \mathrm{O} 2 \ldots \mathrm{O} 1$ hydrogen bonds (Table 1 ), forming an inversionrelated molecule-solvate 2:2 dimer with an $R_{4}^{4}(12)$ ring motif (Fig. 2) (Bernstein et al., 1995). The crystal structure also features $\pi-\pi$ interactions between pairs of inversion-related $(1-x, 1-y, 1-z)$ indolin-2-one rings with an interplanar spacing of 3.599 (2) $\AA$.

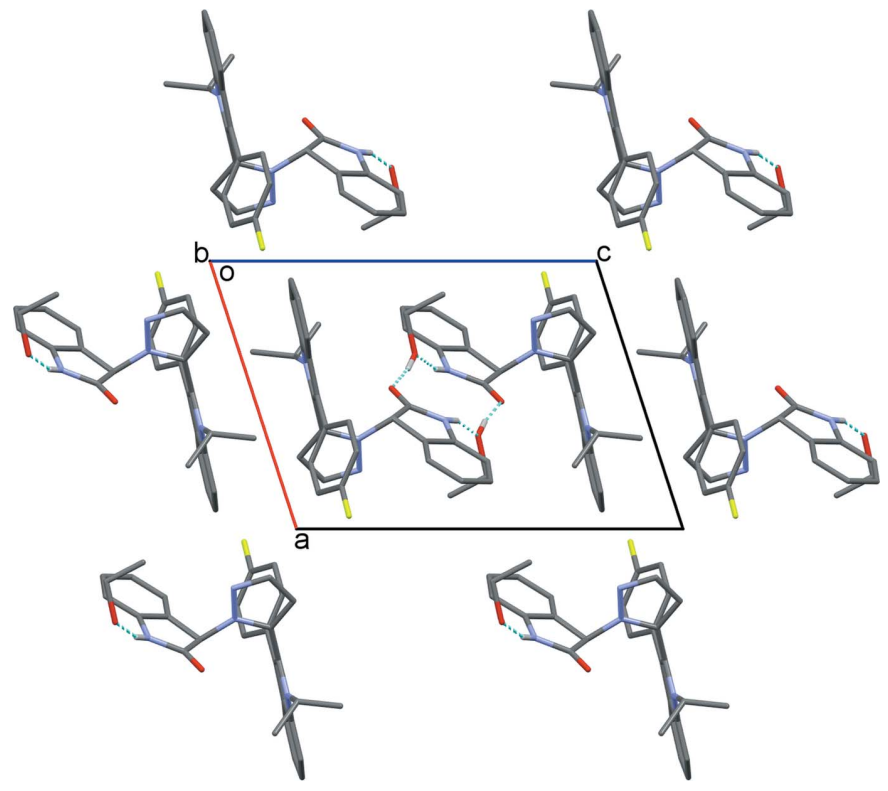

Figure 2

The crystal packing of the title compound viewed along the $b$ axis. The $\mathrm{N}-\mathrm{H} \cdots \mathrm{O}$ and $\mathrm{O}-\mathrm{H} \cdots \mathrm{O}$ hydrogen bonds are shown as dashed lines. $\mathrm{H}$ atoms not involved in hydrogen bonding have been omitted for clarity. 
Table 2

Experimental details.

\begin{tabular}{|c|c|}
\hline \multicolumn{2}{|l|}{ Crystal data } \\
\hline Chemical formula & $\mathrm{C}_{28} \mathrm{H}_{23} \mathrm{FN}_{4} \mathrm{O} \cdot \mathrm{C}_{2} \mathrm{H}_{6} \mathrm{O}$ \\
\hline$M_{\mathrm{r}}$ & 496.57 \\
\hline Crystal system, space group & Triclinic, $P \overline{1}$ \\
\hline Temperature $(\mathrm{K})$ & 297 \\
\hline$a, b, c(\AA)$ & $\begin{array}{l}9.9754(8), 10.2139(8) \\
\quad 14.0294(11)\end{array}$ \\
\hline$\alpha, \beta, \gamma\left(^{\circ}\right)$ & $\begin{array}{l}75.7386(15), 71.0062(14) \\
83.1264(14)\end{array}$ \\
\hline$V\left(\AA^{3}\right)$ & $1308.73(18)$ \\
\hline$Z$ & 2 \\
\hline Radiation type & Мо $K \alpha$ \\
\hline$\mu\left(\mathrm{mm}^{-1}\right)$ & 0.09 \\
\hline Crystal size $(\mathrm{mm})$ & $0.42 \times 0.22 \times 0.22$ \\
\hline \multicolumn{2}{|l|}{ Data collection } \\
\hline Diffractometer & $\begin{array}{l}\text { Bruker APEXII DUO CCD area } \\
\text { detector }\end{array}$ \\
\hline Absorption correction & $\begin{array}{l}\text { Multi-scan (SADABS; Bruker, } \\
\text { 2009) }\end{array}$ \\
\hline$T_{\min }, T_{\max }$ & $0.884,0.955$ \\
\hline $\begin{array}{l}\text { No. of measured, independent and } \\
\text { observed }[I>2 \sigma(I)] \text { reflections }\end{array}$ & $32072,5778,3733$ \\
\hline$R_{\text {int }}$ & 0.032 \\
\hline$(\sin \theta / \lambda)_{\max }\left(\AA^{-1}\right)$ & 0.650 \\
\hline \multicolumn{2}{|l|}{ Refinement } \\
\hline$R\left[F^{2}>2 \sigma\left(F^{2}\right)\right], w R\left(F^{2}\right), S$ & $0.057,0.130,1.21$ \\
\hline No. of reflections & 5778 \\
\hline No. of parameters & 375 \\
\hline No. of restraints & 3 \\
\hline $\mathrm{H}$-atom treatment & $\begin{array}{l}\mathrm{H} \text { atoms treated by a mixture of } \\
\text { independent and constrained } \\
\text { refinement }\end{array}$ \\
\hline$\Delta \rho_{\max }, \Delta \rho_{\min }\left(\mathrm{e} \AA^{-3}\right)$ & $0.15,-0.19$ \\
\hline
\end{tabular}

Computer programs: APEX2 (Bruker, 2009), SAINT (Bruker, 2009), SHELXS97 (Sheldrick, 2008), SHELXL2013 (Sheldrick, 2015), Mercury (Macrae et al., 2008) and PLATON (Spek, 2009).

\section{Database survey}

A search of the Cambridge Structural Database (CSD, Version 35.6, last update May 2015; Groom \& Allen, 2014) using 4-( $\lambda^{1}$-azanyl)-5-methyl-2,4-dihydro-3H-1,2,4-triazole-3thione as the main skeleton, revealed the presence of 57 structures containing the triazole-thione moiety but only four structures containing the fluvastatin nucleus. These include 5-[3-(4-fluorophenyl)-1-isopropyl-1 $H$-indol-2-yl]-1- $(X)$ penta2,4-diene-1-one, where $X=4$-nitrophenyl (NUHNAH), 2-hydroxyphenyl (NUHNEL), 4-methoxyphenyl (NUHNIP) and 4-chlorophenyl (NUHNOV) (Kalalbandi et al., 2015). In these four compounds, the 4-fluorophenyl ring of the fluvastatin nucleus is inclined to the indole ring by dihedral angles ranging from $c a 46.66$ to $68.59^{\circ}$, compared to $40.74(8)^{\circ}$ for the title compound.

\section{Synthesis and crystallization}

The title compound was synthesized by refluxing a hot methanolic solution $(30 \mathrm{~mL})$ of 3-(3-(4-fluorophenyl)-1-isopropylindolin-2-yl)acrylaldehyde $(0.01 \mathrm{~mol})$ and a hot methanolic solution $(30 \mathrm{~mL})$ of 3-hydrazonoeindolin-2-one
$(0.01 \mathrm{~mol})$ for $5 \mathrm{~h}$ with addition of 4 drops of conc. hydrochloric acid (Ajaykumar et al., 2009). The product obtained after evaporation of the solvent was filtered, washed with cold $\mathrm{MeOH}$ and recrystallized from ETOH. The single crystal used for the crystal analysis was grown by the slow evaporation of a solution in chloroform-ethanol (1:1). Yield (m.p.): 78\% $(551 \mathrm{~K}) .{ }^{1} \mathrm{HNMR}\left(\mathrm{CDCl}_{3}\right)$ in p.p.m.: $7.94(s, 1 \mathrm{H}, \mathrm{NH}$, indole), $7.76(d, 1 \mathrm{H}, \mathrm{Ar}-\mathrm{H}), 7.72(m, 2 \mathrm{H}, \mathrm{Ar}-\mathrm{H}), 7.37(m, 2 \mathrm{H}, \mathrm{Ar}-\mathrm{H})$, $7.32(t, 1 \mathrm{H}, \mathrm{Ar}-\mathrm{H}), 7.20(t, 1 \mathrm{H}, \mathrm{Ar}-\mathrm{H}), 7.13(d, 1 \mathrm{H}, \mathrm{Ar}-\mathrm{H}), 7.10$ $(d, 2 \mathrm{H}, \mathrm{Ar}-\mathrm{H}), 6.77(t, 1 \mathrm{H}, \mathrm{Ar}-\mathrm{H}), 6.70(d, 1 \mathrm{H}, \mathrm{Ar}-\mathrm{H}), 6.67(d$, $1 \mathrm{H}$, pyrazole $), 5.48(d, 2 \mathrm{H}$, pyrazole $), 5.37(s, 1 \mathrm{H}$, indole $), 4.73$ ( $m, 1 \mathrm{H}$, isopropyl), $1.73\left(m, 6 \mathrm{H}\right.$, methyl). IR $(\mathrm{KBr}) \mathrm{cm}^{-1}: 3250$ $(\mathrm{N}-\mathrm{H}$, indole $), 2827\left(-\mathrm{CH}_{3}\right), 1720(\mathrm{C}=\mathrm{O}$, ketone $), 1618$ $(\mathrm{C}=\mathrm{C}, \mathrm{Ar}), 1520(\mathrm{C}-\mathrm{C}, \mathrm{Ar}), 1469\left(-\mathrm{CH}_{3}\right), 1221(\mathrm{C}-\mathrm{N})$.

\section{Refinement}

Crystal data, data collection and structure refinement details are summarized in Table 2. The ethanol molecule is disordered over two positions with refined site occupancies of 0.560 (14): 0.440 (14). The disorder components were restrained to have similar geometry. The $\mathrm{N}$-bound $\mathrm{H}$ atom was located in a difference Fourier map and freely refined. The $\mathrm{C}$-bound $\mathrm{H}$ atoms were positioned geometrically $(\mathrm{C}-\mathrm{H}=0.93-0.98 \AA)$ and refined using a riding model with $U_{\text {iso }}(\mathrm{H})=1.5 U_{\text {eq }}(\mathrm{C}$ methyl) and $1.2 U_{\mathrm{eq}}(\mathrm{C})$ for other $\mathrm{H}$ atoms.

\section{Acknowledgements}

This research was supported by a PRGS Research Grant (No. RDU 130121).

\section{References}

Ajaykumar, K., Sangamesh, A. P. \& Prema, S. B. (2009). Int. J. Electrochem. Sci. 4, 717-729.

Archana, V. K. S., Srivastava, V. K. \& Kumar, A. (2002). Eur. J. Med. Chem. 37, 873-882.

Bernstein, J., Davis, R. E., Shimoni, L. \& Chang, N.-L. (1995). Angew. Chem. Int. Ed. Engl. 34, 1555-1573.

Bruker (2009). APEX2, SAINT and SADABS. Bruker AXS Inc., Madison, Wisconsin, USA.

Brzozowski, Z. \& Sączewski, F. (2002). Eur. J. Med. Chem. 37, 709720.

Daisley, R. W. \& Shah, V. K. (1984). J. Pharm. Sci. 73, 407-408.

Elkanzi, N. A. A. (2013). Int. J. Res. Pharm. Biomed. Sci. 4, 17-26.

el-Kashef, H. S., el-Emary, T., Gasquet, M., Timon-David, P., Maldonado, J. \& Vanelle, P. (2000). Pharmazie, 55, 572-576.

Groom, C. R. \& Allen, F. H. (2014). Angew. Chem. Int. Ed. 53, 662671.

Kalalbandi, V. K. A., Seetharamappa, J. \& Katrahalli, U. (2015). RSC Adv. 5, 38748-38759.

Kulkarni, A. D., Rahman, M. L., Mohd. Yusoff, M., Kwong, H. C. \& Quah, C. K. (2015a). Acta Cryst. E71, 1411-1413.

Kulkarni, A. D., Rahman, M. L., Mohd. Yusoff, M., Kwong, H. C. \& Quah, C. K. (2015b). Acta Cryst. E71, 1525-1527.

Macrae, C. F., Bruno, I. J., Chisholm, J. A., Edgington, P. R., McCabe, P., Pidcock, E., Rodriguez-Monge, L., Taylor, R., van de Streek, J. \& Wood, P. A. (2008). J. Appl. Cryst. 41, 466-470.

Mana, S., Pahari, N. \& Sharma, N. K. (2010). The Pharma. Res. 3, 5159. 
Meghashyam, N. N. (2011). J. Chem. Pharm. Res. 3, 38-47.

Pandeya, S. N., Sriram, D., Nath, G. \& DeClercq, E. (1999). Eur. J. Pharm. Sci. 9, 25-31.

Pathak, T. P., Gligorich, K. M., Welm, B. E. \& Sigman, M. S. (2010). J. Am. Chem. Soc. 132, 7870-7871.

Penning, T. D., Khilevich, A., Chen, B. B., Russell, M. A., Boys, M. L., Wang, Y., Duffin, T., Engleman, V. W., Finn, M. B., Freeman, S. K., Hanneke, M. L., Keene, J. L., Klover, J. A., Nickols, G. A., Nickols, M. A., Rader, R. K., Settle, S. L., Shannon, K. E., Steininger, C. N., Westlin, M. M. \& Westlin, W. F. (2006). Bioorg. Med. Chem. Lett. 16, 3156-3161.

Pevarello, P., Fancelli, D., Vulpetti, A., Amici, R., Villa, M., Pittalà, V., Vianello, P., Cameron, A., Ciomei, M., Mercurio, C., Bischoff, J. R., Roletto, F., Varasi, M. \& Brasca, M. G. (2006). Bioorg. Med. Chem. Lett. 16, 1084-1090.
Raju, P. A. G., Mallikarjunarao, R., Gopal, K. V., Sreeramulu, J., Reddy, D. M., Krishnamurthi, K. P. \& Reddy, S. R. (2013). J. Chem. Pharm. Res. 5, 21-27.

Repič, O., Prasad, K. \& Lee, G. T. (2001). Org. Process Res. Dev. 5, 519-527.

Sheldrick, G. M. (2008). Acta Cryst. A64, 112-122.

Sheldrick, G. M. (2015). Acta Cryst. C71, 3-8.

Siddiqui, N., Alam, M. S. \& Ahsan, W. (2008). Acta Pharm. 58, 445454.

Singarave, M. \& Sarkkarai, A. (2011). J. Chem. Pharm. Res. 3, 402413.

Spek, A. L. (2009). Acta Cryst. D65, 148-155.

Taha, M., Moukha-Chafiq, O., Lazrek, H. B., Vasseur, J. \& Imbach, J. (2001). Nucleosides Nucleotides Nucleic Acids, 20, 955-958. 


\section{supporting information}

Acta Cryst. (2016). E72, 283-286 [doi:10.1107/S2056989016001614]

Crystal structure of 3-\{5-[3-(4-fluorophenyl)-1-isopropyl-1H-indol-2-yl]-1Hpyrazol-1-yl\}indolin-2-one ethanol monosolvate

\section{Md. Lutfor Rahman, Ajaykumar D. Kulkarni, Mashitah Mohd. Yusoff, Huey Chong Kwong and Ching Kheng Quah}

Computing details

Data collection: APEX2 (Bruker, 2009); cell refinement: SAINT (Bruker, 2009); data reduction: SAINT (Bruker, 2009); program(s) used to solve structure: SHELXS97 (Sheldrick, 2008); program(s) used to refine structure: SHELXL2013 (Sheldrick, 2015); molecular graphics: SHELXL2013 (Sheldrick, 2015) and Mercury (Macrae et al., 2008); software used to prepare material for publication: SHELXL2013 (Sheldrick, 2015) and PLATON (Spek, 2009).

3-\{5-[3-(4-Fluorophenyl)-1-isopropyl-1H-indol-2-yl]-1H-pyrazol-1-yl\}indolin-2-one ethanol monosolvate

Crystal data

$\mathrm{C}_{28} \mathrm{H}_{23} \mathrm{FN}_{4} \mathrm{O} \cdot \mathrm{C}_{2} \mathrm{H}_{6} \mathrm{O}$

$M_{r}=496.57$

Triclinic, $P \overline{1}$

$a=9.9754(8) \AA$

$b=10.2139(8) \AA$

$c=14.0294(11) \AA$

$\alpha=75.7386(15)^{\circ}$

$\beta=71.0062(14)^{\circ}$

$\gamma=83.1264(14)^{\circ}$

$V=1308.73(18) \AA^{3}$

\section{Data collection}

Bruker APEXII DUO CCD area-detector diffractometer

Radiation source: fine-focus sealed tube Graphite monochromator $\varphi$ and $\omega$ scans

Absorption correction: multi-scan (SADABS; Bruker, 2009)

$T_{\min }=0.884, T_{\max }=0.955$

Refinement

Refinement on $F^{2}$

Least-squares matrix: full

$R\left[F^{2}>2 \sigma\left(F^{2}\right)\right]=0.057$

$w R\left(F^{2}\right)=0.130$

$S=1.21$

5778 reflections
$Z=2$

$F(000)=524$

$D_{\mathrm{x}}=1.260 \mathrm{Mg} \mathrm{m}^{-3}$

Mo $K \alpha$ radiation, $\lambda=0.71073 \AA$

Cell parameters from 9792 reflections

$\theta=2.3-27.6^{\circ}$

$\mu=0.09 \mathrm{~mm}^{-1}$

$T=297 \mathrm{~K}$

Block, colourless

$0.42 \times 0.22 \times 0.22 \mathrm{~mm}$

32072 measured reflections

5778 independent reflections

3733 reflections with $I>2 \sigma(I)$

$R_{\text {int }}=0.032$

$\theta_{\max }=27.5^{\circ}, \theta_{\min }=1.6^{\circ}$

$h=-12 \rightarrow 12$

$k=-13 \rightarrow 13$

$l=-18 \rightarrow 18$

375 parameters

3 restraints

Hydrogen site location: mixed

$\mathrm{H}$ atoms treated by a mixture of independent and constrained refinement 
$w=1 /\left[\sigma^{2}\left(F_{\mathrm{o}}^{2}\right)+(0.0361 P)^{2}+0.3328 P\right]$

where $P=\left(F_{\mathrm{o}}^{2}+2 F_{\mathrm{c}}^{2}\right) / 3$

$(\Delta / \sigma)_{\max }<0.001$

$$
\Delta \rho_{\max }=0.15 \mathrm{e} \AA^{-3}
$$

\section{Special details}

Geometry. All esds (except the esd in the dihedral angle between two 1.s. planes) are estimated using the full covariance matrix. The cell esds are taken into account individually in the estimation of esds in distances, angles and torsion angles; correlations between esds in cell parameters are only used when they are defined by crystal symmetry. An approximate (isotropic) treatment of cell esds is used for estimating esds involving 1.s. planes.

Fractional atomic coordinates and isotropic or equivalent isotropic displacement parameters $\left(\AA^{2}\right)$

\begin{tabular}{|c|c|c|c|c|c|}
\hline & $x$ & $y$ & $z$ & $U_{\text {iso }} * / U_{\text {eq }}$ & Occ. $(<1)$ \\
\hline F1 & $0.95103(15)$ & $0.08376(14)$ & $0.14670(13)$ & $0.0965(5)$ & \\
\hline N1 & $0.37863(16)$ & $0.67252(16)$ & $0.16316(12)$ & $0.0496(4)$ & \\
\hline H1N1 & $0.596(2)$ & $0.795(2)$ & $0.5053(16)$ & $0.059 *$ & \\
\hline N2 & $0.66480(15)$ & $0.68580(15)$ & $0.23475(11)$ & $0.0477(4)$ & \\
\hline N3 & $0.77904(17)$ & 0.76065 (17) & $0.21225(13)$ & $0.0571(4)$ & \\
\hline N4 & $0.5929(2)$ & $0.7342(2)$ & $0.47443(14)$ & $0.0662(5)$ & \\
\hline $\mathrm{O} 1$ & $0.47523(17)$ & $0.85895(18)$ & $0.36321(13)$ & $0.0801(5)$ & \\
\hline $\mathrm{C} 1$ & $0.28456(19)$ & $0.5768(2)$ & $0.17372(14)$ & $0.0500(5)$ & \\
\hline $\mathrm{C} 2$ & $0.1405(2)$ & $0.5909(3)$ & $0.18238(16)$ & $0.0638(6)$ & \\
\hline $\mathrm{H} 2 \mathrm{~A}$ & 0.0924 & 0.6749 & 0.1806 & $0.077 *$ & \\
\hline $\mathrm{C} 3$ & $0.0725(2)$ & 0.4771 & $0.19350(18)$ & $0.0732(7)$ & \\
\hline $\mathrm{H} 3 \mathrm{~A}$ & -0.0236 & 0.4841 & 0.1996 & $0.088^{*}$ & \\
\hline $\mathrm{C} 4$ & $0.1431(2)$ & 0.3511 & 0.19594 (18) & $0.0706(6)$ & \\
\hline $\mathrm{H} 4 \mathrm{~A}$ & 0.0935 & 0.2756 & 0.2038 & $0.085^{*}$ & \\
\hline $\mathrm{C} 5$ & $0.2849(2)$ & $0.3364(2)$ & $0.18689(16)$ & $0.0587(5)$ & \\
\hline H5A & 0.3316 & 0.2518 & 0.1882 & $0.070 *$ & \\
\hline C6 & $0.35815(19)$ & $0.45041(19)$ & $0.17569(13)$ & $0.0468(4)$ & \\
\hline C7 & $0.50280(18)$ & $0.47104(18)$ & $0.16459(13)$ & $0.0436(4)$ & \\
\hline $\mathrm{C} 8$ & 0.62068 (19) & $0.36889(18)$ & $0.16080(14)$ & $0.0452(4)$ & \\
\hline C9 & $0.6011(2)$ & $0.2407(2)$ & $0.22518(16)$ & $0.0565(5)$ & \\
\hline H9A & 0.5122 & 0.2197 & 0.2725 & $0.068 *$ & \\
\hline C10 & $0.7109(2)$ & $0.1441(2)$ & 0.22023 (19) & $0.0671(6)$ & \\
\hline H10A & 0.6964 & 0.0579 & 0.2624 & $0.081 *$ & \\
\hline C11 & $0.8413(2)$ & $0.1780(2)$ & 0.15208 (19) & $0.0640(6)$ & \\
\hline C12 & $0.8657(2)$ & $0.3021(2)$ & $0.08737(18)$ & $0.0613(5)$ & \\
\hline $\mathrm{H} 12 \mathrm{~A}$ & 0.9556 & 0.3225 & 0.0416 & $0.074 *$ & \\
\hline C13 & $0.75482(19)$ & $0.3966(2)$ & $0.09108(16)$ & $0.0522(5)$ & \\
\hline H13A & 0.7699 & 0.4809 & 0.0460 & $0.063 *$ & \\
\hline C14 & $0.51043(18)$ & $0.60667(18)$ & $0.15671(13)$ & $0.0432(4)$ & \\
\hline $\mathrm{C} 15$ & $0.63180(18)$ & $0.68060(17)$ & $0.14957(14)$ & $0.0428(4)$ & \\
\hline $\mathrm{C} 16$ & $0.7318(2)$ & $0.75366(19)$ & $0.06796(15)$ & $0.0547(5)$ & \\
\hline H16A & 0.7398 & 0.7686 & -0.0018 & $0.066^{*}$ & \\
\hline C17 & $0.8187(2)$ & $0.8008(2)$ & $0.11080(16)$ & $0.0549(5)$ & \\
\hline H17A & 0.8957 & 0.8543 & 0.0725 & $0.066^{*}$ & \\
\hline C18 & $0.5866(2)$ & $0.6370(2)$ & $0.34278(14)$ & $0.0502(5)$ & \\
\hline H18A & 0.5028 & 0.5904 & 0.3490 & $0.060 *$ & \\
\hline
\end{tabular}




\begin{tabular}{|c|c|c|c|c|c|}
\hline C19 & $0.6725(2)$ & $0.5487(2)$ & 0.40595 (14) & $0.0523(5)$ & \\
\hline $\mathrm{C} 20$ & $0.7444(2)$ & $0.4273(2)$ & $0.39863(18)$ & $0.0666(6)$ & \\
\hline $\mathrm{H} 20 \mathrm{~A}$ & 0.7423 & 0.3823 & 0.3490 & $0.080^{*}$ & \\
\hline $\mathrm{C} 21$ & $0.8208(3)$ & $0.3727(3)$ & $0.4674(2)$ & $0.0833(7)$ & \\
\hline $\mathrm{H} 21 \mathrm{~A}$ & 0.8715 & 0.2906 & 0.4632 & $0.100^{*}$ & \\
\hline $\mathrm{C} 22$ & $0.8225(3)$ & $0.4381(3)$ & $0.5413(2)$ & $0.0877(8)$ & \\
\hline $\mathrm{H} 22 \mathrm{~A}$ & 0.8746 & 0.3998 & 0.5863 & $0.105^{*}$ & \\
\hline $\mathrm{C} 23$ & $0.7486(3)$ & $0.5592(3)$ & $0.55029(17)$ & $0.0770(7)$ & \\
\hline $\mathrm{H} 23 \mathrm{~A}$ & 0.7489 & 0.6030 & 0.6010 & $0.092 *$ & \\
\hline $\mathrm{C} 24$ & $0.6745(2)$ & $0.6130(2)$ & $0.48173(15)$ & $0.0583(5)$ & \\
\hline $\mathrm{C} 25$ & $0.5419(2)$ & $0.7586(2)$ & $0.39376(17)$ & $0.0593(5)$ & \\
\hline $\mathrm{C} 26$ & $0.3559(2)$ & $0.8204(2)$ & $0.14204(17)$ & $0.0607(5)$ & \\
\hline $\mathrm{H} 26 \mathrm{~A}$ & 0.4430 & 0.8576 & 0.1401 & $0.073^{*}$ & \\
\hline $\mathrm{C} 27$ & $0.2370(3)$ & $0.8682(3)$ & $0.2271(2)$ & $0.0910(8)$ & \\
\hline $\mathrm{H} 27 \mathrm{~A}$ & 0.2350 & 0.9651 & 0.2145 & $0.137^{*}$ & \\
\hline H27B & 0.2526 & 0.8299 & 0.2925 & $0.137^{*}$ & \\
\hline $\mathrm{H} 27 \mathrm{C}$ & 0.1480 & 0.8401 & 0.2281 & $0.137^{*}$ & \\
\hline $\mathrm{C} 28$ & $0.3389(3)$ & $0.8721(3)$ & $0.0352(2)$ & $0.0960(9)$ & \\
\hline $\mathrm{H} 28 \mathrm{~A}$ & 0.3347 & 0.9691 & 0.0190 & $0.144^{*}$ & \\
\hline $\mathrm{H} 28 \mathrm{~B}$ & 0.2529 & 0.8400 & 0.0341 & $0.144^{*}$ & \\
\hline $\mathrm{H} 28 \mathrm{C}$ & 0.4183 & 0.8397 & -0.0149 & $0.144^{*}$ & \\
\hline $\mathrm{O} 2$ & $0.3483(14)$ & $0.060(2)$ & $0.4525(12)$ & $0.115(5)$ & $0.560(14)$ \\
\hline $\mathrm{H} 1 \mathrm{O} 2$ & $0.401(10)$ & $-0.016(8)$ & $0.423(8)$ & $0.138^{*}$ & $0.560(14)$ \\
\hline $\mathrm{C} 29$ & $0.1962(7)$ & $0.0616(11)$ & $0.4632(7)$ & $0.088(2)$ & $0.560(14)$ \\
\hline $\mathrm{H} 29 \mathrm{~A}$ & 0.1654 & 0.1480 & 0.4274 & $0.106^{*}$ & $0.560(14)$ \\
\hline $\mathrm{H} 29 \mathrm{~B}$ & 0.1769 & -0.0096 & 0.4353 & $0.106^{*}$ & $0.560(14)$ \\
\hline $\mathrm{C} 30$ & $0.1250(16)$ & $0.039(2)$ & $0.5740(8)$ & $0.186(9)$ & $0.560(14)$ \\
\hline $\mathrm{H} 30 \mathrm{~A}$ & 0.0243 & 0.0400 & 0.5871 & $0.279 *$ & $0.560(14)$ \\
\hline H30B & 0.1468 & 0.1100 & 0.6001 & $0.279^{*}$ & $0.560(14)$ \\
\hline $\mathrm{H} 30 \mathrm{C}$ & 0.1569 & -0.0464 & 0.6078 & $0.279 *$ & $0.560(14)$ \\
\hline $\mathrm{O} 2 \mathrm{~A}$ & $0.3340(15)$ & $0.080(2)$ & $0.4359(12)$ & $0.077(3)$ & $0.440(14)$ \\
\hline $\mathrm{H} 2 \mathrm{O} 2$ & $0.384(13)$ & $0.024(9)$ & $0.415(9)$ & $0.092 *$ & $0.440(14)$ \\
\hline C29A & $0.2173(11)$ & $-0.0028(13)$ & $0.5152(12)$ & $0.101(4)$ & $0.440(14)$ \\
\hline $\mathrm{H} 29 \mathrm{C}$ & 0.1718 & -0.0491 & 0.4822 & $0.121 *$ & $0.440(14)$ \\
\hline $\mathrm{H} 29 \mathrm{D}$ & 0.2550 & -0.0699 & 0.5632 & $0.121 *$ & $0.440(14)$ \\
\hline $\mathrm{C} 30 \mathrm{~A}$ & $0.1171(11)$ & $0.089(2)$ & $0.5688(13)$ & $0.128(6)$ & $0.440(14)$ \\
\hline $\mathrm{H} 30 \mathrm{D}$ & 0.0319 & 0.0425 & 0.6102 & $0.192 *$ & $0.440(14)$ \\
\hline $\mathrm{H} 30 \mathrm{E}$ & 0.0953 & 0.1644 & 0.5194 & $0.192 *$ & $0.440(14)$ \\
\hline $\mathrm{H} 30 \mathrm{~F}$ & 0.1573 & 0.1195 & 0.6124 & $0.192 *$ & $0.440(14)$ \\
\hline
\end{tabular}

Atomic displacement parameters $\left(\AA^{2}\right)$

\begin{tabular}{lllllll}
\hline & $U^{11}$ & $U^{22}$ & $U^{33}$ & $U^{12}$ & $U^{13}$ & $U^{23}$ \\
\hline F1 & $0.0752(9)$ & $0.0731(9)$ & $0.1446(14)$ & $0.0267(7)$ & $-0.0456(9)$ & $-0.0283(9)$ \\
N1 & $0.0461(9)$ & $0.0524(9)$ & $0.0552(9)$ & $0.0063(8)$ & $-0.0226(7)$ & $-0.0154(7)$ \\
N2 & $0.0442(9)$ & $0.0535(9)$ & $0.0478(9)$ & $-0.0089(7)$ & $-0.0156(7)$ & $-0.0103(7)$ \\
N3 & $0.0507(10)$ & $0.0629(11)$ & $0.0610(11)$ & $-0.0173(8)$ & $-0.0171(8)$ & $-0.0129(8)$ \\
N4 & $0.0666(12)$ & $0.0836(14)$ & $0.0578(11)$ & $-0.0107(10)$ & $-0.0164(9)$ & $-0.0328(10)$
\end{tabular}




\begin{tabular}{|c|c|c|c|c|c|c|}
\hline $\mathrm{O} 1$ & $0.0743(11)$ & $0.0801(11)$ & $0.0922(12)$ & $0.0141(9)$ & $-0.0286(9)$ & $-0.0347(10)$ \\
\hline $\mathrm{C} 1$ & $0.0439(11)$ & $0.0653(12)$ & $0.0441(10)$ & $0.0005(9)$ & $-0.0173(8)$ & $-0.0139(9)$ \\
\hline $\mathrm{C} 2$ & $0.0469(12)$ & $0.0835(16)$ & $0.0632(13)$ & $0.0037(11)$ & $-0.0217(10)$ & $-0.0167(11)$ \\
\hline $\mathrm{C} 3$ & $0.0428(12)$ & $0.110(2)$ & $0.0685(15)$ & $-0.0097(13)$ & $-0.0182(11)$ & $-0.0177(14)$ \\
\hline $\mathrm{C} 4$ & $0.0574(14)$ & 0.0905 (18) & $0.0691(15)$ & $-0.0241(13)$ & $-0.0209(11)$ & $-0.0151(12)$ \\
\hline C5 & $0.0551(12)$ & $0.0682(13)$ & $0.0579(12)$ & $-0.0115(10)$ & $-0.0187(10)$ & $-0.0172(10)$ \\
\hline C6 & $0.0435(10)$ & $0.0590(12)$ & $0.0415(10)$ & $-0.0048(9)$ & $-0.0152(8)$ & $-0.0136(8)$ \\
\hline $\mathrm{C} 7$ & $0.0439(10)$ & $0.0496(10)$ & $0.0415(10)$ & $-0.0010(8)$ & $-0.0165(8)$ & $-0.0139(8)$ \\
\hline $\mathrm{C} 8$ & $0.0456(10)$ & $0.0481(11)$ & $0.0489(11)$ & $-0.0003(8)$ & $-0.0200(8)$ & $-0.0168(9)$ \\
\hline C9 & $0.0552(12)$ & $0.0539(12)$ & $0.0599(12)$ & $-0.0033(10)$ & $-0.0186(10)$ & $-0.0103(10)$ \\
\hline $\mathrm{C} 10$ & $0.0733(15)$ & $0.0502(12)$ & $0.0804(16)$ & $0.0023(11)$ & $-0.0345(13)$ & $-0.0066(11)$ \\
\hline $\mathrm{C} 11$ & $0.0553(13)$ & $0.0572(13)$ & 0.0909 (17) & $0.0167(11)$ & $-0.0365(12)$ & $-0.0273(12)$ \\
\hline $\mathrm{C} 12$ & $0.0465(12)$ & $0.0613(13)$ & $0.0792(15)$ & $0.0015(10)$ & $-0.0182(10)$ & $-0.0244(12)$ \\
\hline $\mathrm{C} 13$ & $0.0480(11)$ & $0.0476(11)$ & $0.0623(12)$ & $-0.0003(9)$ & $-0.0169(9)$ & $-0.0156(9)$ \\
\hline $\mathrm{C} 14$ & $0.0428(10)$ & $0.0486(10)$ & $0.0417(10)$ & $0.0023(8)$ & $-0.0172(8)$ & $-0.0126(8)$ \\
\hline $\mathrm{C} 15$ & $0.0440(10)$ & $0.0412(10)$ & $0.0469(10)$ & $0.0044(8)$ & $-0.0189(8)$ & $-0.0131(8)$ \\
\hline $\mathrm{C} 16$ & $0.0605(12)$ & $0.0545(12)$ & $0.0474(11)$ & $-0.0048(10)$ & $-0.0153(10)$ & $-0.0085(9)$ \\
\hline $\mathrm{C} 17$ & $0.0509(11)$ & $0.0497(11)$ & $0.0587(13)$ & $-0.0071(9)$ & $-0.0102(10)$ & $-0.0091(9)$ \\
\hline $\mathrm{C} 18$ & $0.0468(11)$ & $0.0604(12)$ & $0.0463(11)$ & $-0.0129(9)$ & $-0.0132(9)$ & $-0.0133(9)$ \\
\hline $\mathrm{C} 19$ & $0.0498(11)$ & $0.0611(12)$ & $0.0459(11)$ & $-0.0156(10)$ & $-0.0143(9)$ & $-0.0056(9)$ \\
\hline $\mathrm{C} 20$ & $0.0711(14)$ & $0.0623(14)$ & $0.0647(14)$ & $-0.0098(12)$ & $-0.0221(12)$ & $-0.0062(11)$ \\
\hline $\mathrm{C} 21$ & $0.0802(17)$ & $0.0738(16)$ & $0.0876(19)$ & $-0.0054(13)$ & $-0.0323(15)$ & $0.0069(14)$ \\
\hline $\mathrm{C} 22$ & $0.0842(18)$ & $0.106(2)$ & 0.0709 (17) & $-0.0246(17)$ & $-0.0405(14)$ & $0.0165(16)$ \\
\hline $\mathrm{C} 23$ & $0.0804(17)$ & $0.104(2)$ & $0.0514(13)$ & $-0.0295(15)$ & $-0.0261(12)$ & $-0.0049(13)$ \\
\hline $\mathrm{C} 24$ & $0.0553(12)$ & $0.0755(15)$ & $0.0457(11)$ & $-0.0182(11)$ & $-0.0141(9)$ & $-0.0107(10)$ \\
\hline $\mathrm{C} 25$ & $0.0486(12)$ & $0.0704(14)$ & $0.0597(13)$ & $-0.0066(11)$ & $-0.0112(10)$ & $-0.0215(11)$ \\
\hline $\mathrm{C} 26$ & $0.0644(13)$ & $0.0514(12)$ & $0.0739(14)$ & $0.0118(10)$ & $-0.0336(11)$ & $-0.0173(10)$ \\
\hline $\mathrm{C} 27$ & $0.0857(18)$ & $0.0839(18)$ & $0.113(2)$ & $0.0301(15)$ & $-0.0360(16)$ & $-0.0465(16)$ \\
\hline $\mathrm{C} 28$ & $0.133(2)$ & $0.0728(17)$ & $0.090(2)$ & $0.0009(16)$ & $-0.0625(19)$ & $0.0015(14)$ \\
\hline $\mathrm{O} 2$ & $0.070(5)$ & $0.111(9)$ & $0.192(12)$ & $0.006(4)$ & $-0.033(6)$ & $-0.099(9)$ \\
\hline $\mathrm{C} 29$ & $0.081(5)$ & $0.095(5)$ & $0.094(5)$ & $-0.007(3)$ & $-0.031(4)$ & $-0.025(4)$ \\
\hline $\mathrm{C} 30$ & $0.175(13)$ & $0.31(2)$ & $0.089(7)$ & $-0.110(12)$ & $-0.025(7)$ & $-0.046(9)$ \\
\hline $\mathrm{O} 2 \mathrm{~A}$ & $0.064(7)$ & $0.082(5)$ & $0.085(4)$ & $-0.007(5)$ & $-0.006(4)$ & $-0.041(3)$ \\
\hline C29A & $0.089(7)$ & $0.106(8)$ & $0.109(9)$ & $-0.013(5)$ & $-0.018(6)$ & $-0.038(7)$ \\
\hline $\mathrm{C} 30 \mathrm{~A}$ & $0.041(5)$ & $0.148(9)$ & $0.186(14)$ & $-0.015(5)$ & $0.006(6)$ & $-0.071(8)$ \\
\hline
\end{tabular}

Geometric parameters $\left(\AA,{ }^{o}\right)$

\begin{tabular}{llll}
\hline $\mathrm{F} 1-\mathrm{C} 11$ & $1.364(2)$ & $\mathrm{C} 17-\mathrm{H} 17 \mathrm{~A}$ & 0.9300 \\
$\mathrm{~N} 1-\mathrm{C} 1$ & $1.384(2)$ & $\mathrm{C} 18-\mathrm{C} 19$ & $1.500(3)$ \\
$\mathrm{N} 1-\mathrm{C} 14$ & $1.389(2)$ & $\mathrm{C} 18-\mathrm{C} 25$ & $1.532(3)$ \\
$\mathrm{N} 1-\mathrm{C} 26$ & $1.470(2)$ & $\mathrm{C} 18-\mathrm{H} 18 \mathrm{~A}$ & 0.9800 \\
$\mathrm{~N} 2-\mathrm{C} 15$ & $1.353(2)$ & $\mathrm{C} 19-\mathrm{C} 20$ & $1.365(3)$ \\
$\mathrm{N} 2-\mathrm{N} 3$ & $1.356(2)$ & $\mathrm{C} 19-\mathrm{C} 24$ & $1.386(3)$ \\
$\mathrm{N} 2-\mathrm{C} 18$ & $1.451(2)$ & $\mathrm{C} 20-\mathrm{C} 21$ & $1.391(3)$ \\
$\mathrm{N} 3-\mathrm{C} 17$ & $1.317(2)$ & $\mathrm{C} 20-\mathrm{H} 20 \mathrm{~A}$ & 0.9300 \\
$\mathrm{~N} 4-\mathrm{C} 25$ & $1.344(3)$ & $\mathrm{C} 21-\mathrm{C} 22$ & $1.370(4)$ \\
$\mathrm{N} 4-\mathrm{C} 24$ & $1.399(3)$ & $\mathrm{C} 21-\mathrm{H} 21 \mathrm{~A}$ & 0.9300
\end{tabular}




\begin{tabular}{|c|c|c|c|}
\hline $\mathrm{N} 4-\mathrm{H} 1 \mathrm{~N} 1$ & $0.85(2)$ & $\mathrm{C} 22-\mathrm{C} 23$ & $1.374(4)$ \\
\hline $\mathrm{O} 1-\mathrm{C} 25$ & $1.218(3)$ & $\mathrm{C} 22-\mathrm{H} 22 \mathrm{~A}$ & 0.9300 \\
\hline $\mathrm{C} 1-\mathrm{C} 2$ & $1.395(3)$ & $\mathrm{C} 23-\mathrm{C} 24$ & $1.371(3)$ \\
\hline $\mathrm{C} 1-\mathrm{C} 6$ & $1.406(3)$ & $\mathrm{C} 23-\mathrm{H} 23 \mathrm{~A}$ & 0.9300 \\
\hline $\mathrm{C} 2-\mathrm{C} 3$ & $1.367(3)$ & $\mathrm{C} 26-\mathrm{C} 27$ & $1.515(3)$ \\
\hline $\mathrm{C} 2-\mathrm{H} 2 \mathrm{~A}$ & 0.9300 & $\mathrm{C} 26-\mathrm{C} 28$ & $1.519(3)$ \\
\hline $\mathrm{C} 3-\mathrm{C} 4$ & $1.389(3)$ & $\mathrm{C} 26-\mathrm{H} 26 \mathrm{~A}$ & 0.9800 \\
\hline $\mathrm{C} 3-\mathrm{H} 3 \mathrm{~A}$ & 0.9300 & $\mathrm{C} 27-\mathrm{H} 27 \mathrm{~A}$ & 0.9600 \\
\hline $\mathrm{C} 4-\mathrm{C} 5$ & $1.371(3)$ & C27-H27B & 0.9600 \\
\hline $\mathrm{C} 4-\mathrm{H} 4 \mathrm{~A}$ & 0.9300 & $\mathrm{C} 27-\mathrm{H} 27 \mathrm{C}$ & 0.9600 \\
\hline $\mathrm{C} 5-\mathrm{C} 6$ & $1.398(3)$ & $\mathrm{C} 28-\mathrm{H} 28 \mathrm{~A}$ & 0.9600 \\
\hline $\mathrm{C} 5-\mathrm{H} 5 \mathrm{~A}$ & 0.9300 & $\mathrm{C} 28-\mathrm{H} 28 \mathrm{~B}$ & 0.9600 \\
\hline $\mathrm{C} 6-\mathrm{C} 7$ & $1.436(2)$ & $\mathrm{C} 28-\mathrm{H} 28 \mathrm{C}$ & 0.9600 \\
\hline $\mathrm{C} 7-\mathrm{C} 14$ & $1.372(2)$ & $\mathrm{O} 2-\mathrm{C} 29$ & $1.474(13)$ \\
\hline $\mathrm{C} 7-\mathrm{C} 8$ & $1.472(2)$ & $\mathrm{O} 2-\mathrm{H} 1 \mathrm{O} 2$ & $0.99(9)$ \\
\hline $\mathrm{C} 8-\mathrm{C} 13$ & $1.389(3)$ & $\mathrm{C} 29-\mathrm{C} 30$ & $1.456(12)$ \\
\hline $\mathrm{C} 8-\mathrm{C} 9$ & $1.390(3)$ & $\mathrm{C} 29-\mathrm{H} 29 \mathrm{~A}$ & 0.9700 \\
\hline $\mathrm{C} 9-\mathrm{C} 10$ & $1.380(3)$ & $\mathrm{C} 29-\mathrm{H} 29 \mathrm{~B}$ & 0.9700 \\
\hline $\mathrm{C} 9-\mathrm{H} 9 \mathrm{~A}$ & 0.9300 & $\mathrm{C} 30-\mathrm{H} 30 \mathrm{~A}$ & 0.9600 \\
\hline $\mathrm{C} 10-\mathrm{C} 11$ & $1.364(3)$ & $\mathrm{C} 30-\mathrm{H} 30 \mathrm{~B}$ & 0.9600 \\
\hline $\mathrm{C} 10-\mathrm{H} 10 \mathrm{~A}$ & 0.9300 & $\mathrm{C} 30-\mathrm{H} 30 \mathrm{C}$ & 0.9600 \\
\hline $\mathrm{C} 11-\mathrm{C} 12$ & $1.361(3)$ & $\mathrm{O} 2 \mathrm{~A}-\mathrm{C} 29 \mathrm{~A}$ & $1.497(13)$ \\
\hline $\mathrm{C} 12-\mathrm{C} 13$ & $1.375(3)$ & $\mathrm{O} 2 \mathrm{~A}-\mathrm{H} 2 \mathrm{O} 2$ & $0.76(10)$ \\
\hline $\mathrm{C} 12-\mathrm{H} 12 \mathrm{~A}$ & 0.9300 & $\mathrm{C} 29 \mathrm{~A}-\mathrm{C} 30 \mathrm{~A}$ & $1.438(14)$ \\
\hline $\mathrm{C} 13-\mathrm{H} 13 \mathrm{~A}$ & 0.9300 & $\mathrm{C} 29 \mathrm{~A}-\mathrm{H} 29 \mathrm{C}$ & 0.9700 \\
\hline $\mathrm{C} 14-\mathrm{C} 15$ & $1.466(2)$ & $\mathrm{C} 29 \mathrm{~A}-\mathrm{H} 29 \mathrm{D}$ & 0.9700 \\
\hline $\mathrm{C} 15-\mathrm{C} 16$ & $1.370(3)$ & $\mathrm{C} 30 \mathrm{~A}-\mathrm{H} 30 \mathrm{D}$ & 0.9600 \\
\hline $\mathrm{C} 16-\mathrm{C} 17$ & $1.388(3)$ & $\mathrm{C} 30 \mathrm{~A}-\mathrm{H} 30 \mathrm{E}$ & 0.9600 \\
\hline $\mathrm{C} 16-\mathrm{H} 16 \mathrm{~A}$ & 0.9300 & $\mathrm{C} 30 \mathrm{~A}-\mathrm{H} 30 \mathrm{~F}$ & 0.9600 \\
\hline $\mathrm{C} 1-\mathrm{N} 1-\mathrm{C} 14$ & $107.55(15)$ & $\mathrm{C} 25-\mathrm{C} 18-\mathrm{H} 18 \mathrm{~A}$ & 110.2 \\
\hline $\mathrm{C} 1-\mathrm{N} 1-\mathrm{C} 26$ & $127.68(16)$ & $\mathrm{C} 20-\mathrm{C} 19-\mathrm{C} 24$ & $120.1(2)$ \\
\hline $\mathrm{C} 14-\mathrm{N} 1-\mathrm{C} 26$ & $123.80(16)$ & $\mathrm{C} 20-\mathrm{C} 19-\mathrm{C} 18$ & $131.91(19)$ \\
\hline $\mathrm{C} 15-\mathrm{N} 2-\mathrm{N} 3$ & $112.64(15)$ & $\mathrm{C} 24-\mathrm{C} 19-\mathrm{C} 18$ & $107.96(18)$ \\
\hline $\mathrm{C} 15-\mathrm{N} 2-\mathrm{C} 18$ & $129.00(15)$ & $\mathrm{C} 19-\mathrm{C} 20-\mathrm{C} 21$ & $118.2(2)$ \\
\hline $\mathrm{N} 3-\mathrm{N} 2-\mathrm{C} 18$ & $117.92(15)$ & $\mathrm{C} 19-\mathrm{C} 20-\mathrm{H} 20 \mathrm{~A}$ & 120.9 \\
\hline $\mathrm{C} 17-\mathrm{N} 3-\mathrm{N} 2$ & $104.16(15)$ & $\mathrm{C} 21-\mathrm{C} 20-\mathrm{H} 20 \mathrm{~A}$ & 120.9 \\
\hline $\mathrm{C} 25-\mathrm{N} 4-\mathrm{C} 24$ & $111.97(18)$ & $\mathrm{C} 22-\mathrm{C} 21-\mathrm{C} 20$ & $120.9(3)$ \\
\hline $\mathrm{C} 25-\mathrm{N} 4-\mathrm{H} 1 \mathrm{~N} 1$ & $122.7(14)$ & $\mathrm{C} 22-\mathrm{C} 21-\mathrm{H} 21 \mathrm{~A}$ & 119.5 \\
\hline $\mathrm{C} 24-\mathrm{N} 4-\mathrm{H} 1 \mathrm{~N} 1$ & $122.9(14)$ & $\mathrm{C} 20-\mathrm{C} 21-\mathrm{H} 21 \mathrm{~A}$ & 119.5 \\
\hline $\mathrm{N} 1-\mathrm{C} 1-\mathrm{C} 2$ & $130.23(19)$ & $\mathrm{C} 21-\mathrm{C} 22-\mathrm{C} 23$ & $121.3(2)$ \\
\hline $\mathrm{N} 1-\mathrm{C} 1-\mathrm{C} 6$ & $108.26(15)$ & $\mathrm{C} 21-\mathrm{C} 22-\mathrm{H} 22 \mathrm{~A}$ & 119.4 \\
\hline $\mathrm{C} 2-\mathrm{C} 1-\mathrm{C} 6$ & $121.50(19)$ & $\mathrm{C} 23-\mathrm{C} 22-\mathrm{H} 22 \mathrm{~A}$ & 119.4 \\
\hline $\mathrm{C} 3-\mathrm{C} 2-\mathrm{C} 1$ & $117.7(2)$ & $\mathrm{C} 24-\mathrm{C} 23-\mathrm{C} 22$ & $117.5(2)$ \\
\hline $\mathrm{C} 3-\mathrm{C} 2-\mathrm{H} 2 \mathrm{~A}$ & 121.2 & $\mathrm{C} 24-\mathrm{C} 23-\mathrm{H} 23 \mathrm{~A}$ & 121.3 \\
\hline $\mathrm{C} 1-\mathrm{C} 2-\mathrm{H} 2 \mathrm{~A}$ & 121.2 & $\mathrm{C} 22-\mathrm{C} 23-\mathrm{H} 23 \mathrm{~A}$ & 121.3 \\
\hline $\mathrm{C} 2-\mathrm{C} 3-\mathrm{C} 4$ & $121.7(2)$ & $\mathrm{C} 23-\mathrm{C} 24-\mathrm{C} 19$ & $122.0(2)$ \\
\hline $\mathrm{C} 2-\mathrm{C} 3-\mathrm{H} 3 \mathrm{~A}$ & 119.1 & $\mathrm{C} 23-\mathrm{C} 24-\mathrm{N} 4$ & $128.3(2)$ \\
\hline
\end{tabular}




\begin{tabular}{|c|c|}
\hline $\mathrm{C} 4-\mathrm{C} 3-\mathrm{H} 3 \mathrm{~A}$ & 119.1 \\
\hline $\mathrm{C} 5-\mathrm{C} 4-\mathrm{C} 3$ & $121.0(2)$ \\
\hline $\mathrm{C} 5-\mathrm{C} 4-\mathrm{H} 4 \mathrm{~A}$ & 119.5 \\
\hline $\mathrm{C} 3-\mathrm{C} 4-\mathrm{H} 4 \mathrm{~A}$ & 119.5 \\
\hline $\mathrm{C} 4-\mathrm{C} 5-\mathrm{C} 6$ & $119.0(2)$ \\
\hline $\mathrm{C} 4-\mathrm{C} 5-\mathrm{H} 5 \mathrm{~A}$ & 120.5 \\
\hline $\mathrm{C} 6-\mathrm{C} 5-\mathrm{H} 5 \mathrm{~A}$ & 120.5 \\
\hline $\mathrm{C} 5-\mathrm{C} 6-\mathrm{C} 1$ & $119.09(17)$ \\
\hline $\mathrm{C} 5-\mathrm{C} 6-\mathrm{C} 7$ & $133.46(18)$ \\
\hline $\mathrm{C} 1-\mathrm{C} 6-\mathrm{C} 7$ & $107.45(16)$ \\
\hline $\mathrm{C} 14-\mathrm{C} 7-\mathrm{C} 6$ & $106.08(15)$ \\
\hline $\mathrm{C} 14-\mathrm{C} 7-\mathrm{C} 8$ & $126.39(16)$ \\
\hline $\mathrm{C} 6-\mathrm{C} 7-\mathrm{C} 8$ & $127.53(16)$ \\
\hline $\mathrm{C} 13-\mathrm{C} 8-\mathrm{C} 9$ & $117.72(17)$ \\
\hline $\mathrm{C} 13-\mathrm{C} 8-\mathrm{C} 7$ & $121.00(17)$ \\
\hline $\mathrm{C} 9-\mathrm{C} 8-\mathrm{C} 7$ & $121.27(17)$ \\
\hline $\mathrm{C} 10-\mathrm{C} 9-\mathrm{C} 8$ & $121.3(2)$ \\
\hline $\mathrm{C} 10-\mathrm{C} 9-\mathrm{H} 9 \mathrm{~A}$ & 119.4 \\
\hline C8-C9-H9A & 119.4 \\
\hline $\mathrm{C} 11-\mathrm{C} 10-\mathrm{C} 9$ & $118.5(2)$ \\
\hline $\mathrm{C} 11-\mathrm{C} 10-\mathrm{H} 10 \mathrm{~A}$ & 120.8 \\
\hline $\mathrm{C} 9-\mathrm{C} 10-\mathrm{H} 10 \mathrm{~A}$ & 120.8 \\
\hline $\mathrm{C} 12-\mathrm{C} 11-\mathrm{F} 1$ & $118.5(2)$ \\
\hline $\mathrm{C} 12-\mathrm{C} 11-\mathrm{C} 10$ & $122.39(19)$ \\
\hline $\mathrm{F} 1-\mathrm{C} 11-\mathrm{C} 10$ & $119.1(2)$ \\
\hline $\mathrm{C} 11-\mathrm{C} 12-\mathrm{C} 13$ & $118.7(2)$ \\
\hline $\mathrm{C} 11-\mathrm{C} 12-\mathrm{H} 12 \mathrm{~A}$ & 120.7 \\
\hline $\mathrm{C} 13-\mathrm{C} 12-\mathrm{H} 12 \mathrm{~A}$ & 120.7 \\
\hline $\mathrm{C} 12-\mathrm{C} 13-\mathrm{C} 8$ & $121.40(19)$ \\
\hline $\mathrm{C} 12-\mathrm{C} 13-\mathrm{H} 13 \mathrm{~A}$ & 119.3 \\
\hline $\mathrm{C} 8-\mathrm{C} 13-\mathrm{H} 13 \mathrm{~A}$ & 119.3 \\
\hline $\mathrm{C} 7-\mathrm{C} 14-\mathrm{N} 1$ & $110.64(16)$ \\
\hline $\mathrm{C} 7-\mathrm{C} 14-\mathrm{C} 15$ & $128.66(16)$ \\
\hline $\mathrm{N} 1-\mathrm{C} 14-\mathrm{C} 15$ & $120.57(15)$ \\
\hline $\mathrm{N} 2-\mathrm{C} 15-\mathrm{C} 16$ & $105.47(16)$ \\
\hline $\mathrm{N} 2-\mathrm{C} 15-\mathrm{C} 14$ & $121.55(16)$ \\
\hline $\mathrm{C} 16-\mathrm{C} 15-\mathrm{C} 14$ & $132.98(17)$ \\
\hline $\mathrm{C} 15-\mathrm{C} 16-\mathrm{C} 17$ & $105.79(18)$ \\
\hline $\mathrm{C} 15-\mathrm{C} 16-\mathrm{H} 16 \mathrm{~A}$ & 127.1 \\
\hline $\mathrm{C} 17-\mathrm{C} 16-\mathrm{H} 16 \mathrm{~A}$ & 127.1 \\
\hline $\mathrm{N} 3-\mathrm{C} 17-\mathrm{C} 16$ & $111.93(18)$ \\
\hline N3-C17-H17A & 124.0 \\
\hline $\mathrm{C} 16-\mathrm{C} 17-\mathrm{H} 17 \mathrm{~A}$ & 124.0 \\
\hline $\mathrm{N} 2-\mathrm{C} 18-\mathrm{C} 19$ & $114.71(15)$ \\
\hline $\mathrm{N} 2-\mathrm{C} 18-\mathrm{C} 25$ & $108.29(16)$ \\
\hline $\mathrm{C} 19-\mathrm{C} 18-\mathrm{C} 25$ & $102.81(16)$ \\
\hline $\mathrm{N} 2-\mathrm{C} 18-\mathrm{H} 18 \mathrm{~A}$ & 110.2 \\
\hline $\mathrm{C} 19-\mathrm{C} 18-\mathrm{H} 18 \mathrm{~A}$ & 110.2 \\
\hline
\end{tabular}

\begin{tabular}{|c|c|}
\hline $\mathrm{C} 19-\mathrm{C} 24-\mathrm{N} 4$ & $109.66(18)$ \\
\hline $\mathrm{O} 1-\mathrm{C} 25-\mathrm{N} 4$ & $127.7(2)$ \\
\hline $\mathrm{O} 1-\mathrm{C} 25-\mathrm{C} 18$ & $124.8(2)$ \\
\hline $\mathrm{N} 4-\mathrm{C} 25-\mathrm{C} 18$ & $107.5(2)$ \\
\hline $\mathrm{N} 1-\mathrm{C} 26-\mathrm{C} 27$ & $112.74(19)$ \\
\hline $\mathrm{N} 1-\mathrm{C} 26-\mathrm{C} 28$ & $110.06(18)$ \\
\hline $\mathrm{C} 27-\mathrm{C} 26-\mathrm{C} 28$ & $113.7(2)$ \\
\hline $\mathrm{N} 1-\mathrm{C} 26-\mathrm{H} 26 \mathrm{~A}$ & 106.6 \\
\hline $\mathrm{C} 27-\mathrm{C} 26-\mathrm{H} 26 \mathrm{~A}$ & 106.6 \\
\hline $\mathrm{C} 28-\mathrm{C} 26-\mathrm{H} 26 \mathrm{~A}$ & 106.6 \\
\hline $\mathrm{C} 26-\mathrm{C} 27-\mathrm{H} 27 \mathrm{~A}$ & 109.5 \\
\hline $\mathrm{C} 26-\mathrm{C} 27-\mathrm{H} 27 \mathrm{~B}$ & 109.5 \\
\hline $\mathrm{H} 27 \mathrm{~A}-\mathrm{C} 27-\mathrm{H} 27 \mathrm{~B}$ & 109.5 \\
\hline $\mathrm{C} 26-\mathrm{C} 27-\mathrm{H} 27 \mathrm{C}$ & 109.5 \\
\hline $\mathrm{H} 27 \mathrm{~A}-\mathrm{C} 27-\mathrm{H} 27 \mathrm{C}$ & 109.5 \\
\hline $\mathrm{H} 27 \mathrm{~B}-\mathrm{C} 27-\mathrm{H} 27 \mathrm{C}$ & 109.5 \\
\hline $\mathrm{C} 26-\mathrm{C} 28-\mathrm{H} 28 \mathrm{~A}$ & 109.5 \\
\hline $\mathrm{C} 26-\mathrm{C} 28-\mathrm{H} 28 \mathrm{~B}$ & 109.5 \\
\hline $\mathrm{H} 28 \mathrm{~A}-\mathrm{C} 28-\mathrm{H} 28 \mathrm{~B}$ & 109.5 \\
\hline $\mathrm{C} 26-\mathrm{C} 28-\mathrm{H} 28 \mathrm{C}$ & 109.5 \\
\hline $\mathrm{H} 28 \mathrm{~A}-\mathrm{C} 28-\mathrm{H} 28 \mathrm{C}$ & 109.5 \\
\hline $\mathrm{H} 28 \mathrm{~B}-\mathrm{C} 28-\mathrm{H} 28 \mathrm{C}$ & 109.5 \\
\hline $\mathrm{C} 29-\mathrm{O} 2-\mathrm{H} 1 \mathrm{O} 2$ & $111(6)$ \\
\hline $\mathrm{C} 30-\mathrm{C} 29-\mathrm{O} 2$ & $104.6(11)$ \\
\hline $\mathrm{C} 30-\mathrm{C} 29-\mathrm{H} 29 \mathrm{~A}$ & 110.8 \\
\hline $\mathrm{O} 2-\mathrm{C} 29-\mathrm{H} 29 \mathrm{~A}$ & 110.8 \\
\hline $\mathrm{C} 30-\mathrm{C} 29-\mathrm{H} 29 \mathrm{~B}$ & 110.8 \\
\hline $\mathrm{O} 2-\mathrm{C} 29-\mathrm{H} 29 \mathrm{~B}$ & 110.8 \\
\hline $\mathrm{H} 29 \mathrm{~A}-\mathrm{C} 29-\mathrm{H} 29 \mathrm{~B}$ & 108.9 \\
\hline $\mathrm{C} 29-\mathrm{C} 30-\mathrm{H} 30 \mathrm{~A}$ & 109.5 \\
\hline $\mathrm{C} 29-\mathrm{C} 30-\mathrm{H} 30 \mathrm{~B}$ & 109.5 \\
\hline $\mathrm{H} 30 \mathrm{~A}-\mathrm{C} 30-\mathrm{H} 30 \mathrm{~B}$ & 109.5 \\
\hline C29-C30-H30C & 109.5 \\
\hline $\mathrm{H} 30 \mathrm{~A}-\mathrm{C} 30-\mathrm{H} 30 \mathrm{C}$ & 109.5 \\
\hline $\mathrm{H} 30 \mathrm{~B}-\mathrm{C} 30-\mathrm{H} 30 \mathrm{C}$ & 109.5 \\
\hline $\mathrm{C} 29 \mathrm{~A}-\mathrm{O} 2 \mathrm{~A}-\mathrm{H} 2 \mathrm{O} 2$ & $99(10)$ \\
\hline $\mathrm{C} 30 \mathrm{~A}-\mathrm{C} 29 \mathrm{~A}-\mathrm{O} 2 \mathrm{~A}$ & $107.1(13)$ \\
\hline $\mathrm{C} 30 \mathrm{~A}-\mathrm{C} 29 \mathrm{~A}-\mathrm{H} 29 \mathrm{C}$ & 110.3 \\
\hline $\mathrm{O} 2 \mathrm{~A}-\mathrm{C} 29 \mathrm{~A}-\mathrm{H} 29 \mathrm{C}$ & 110.3 \\
\hline $\mathrm{C} 30 \mathrm{~A}-\mathrm{C} 29 \mathrm{~A}-\mathrm{H} 29 \mathrm{D}$ & 110.3 \\
\hline $\mathrm{O} 2 \mathrm{~A}-\mathrm{C} 29 \mathrm{~A}-\mathrm{H} 29 \mathrm{D}$ & 110.3 \\
\hline $\mathrm{H} 29 \mathrm{C}-\mathrm{C} 29 \mathrm{~A}-\mathrm{H} 29 \mathrm{D}$ & 108.5 \\
\hline $\mathrm{C} 29 \mathrm{~A}-\mathrm{C} 30 \mathrm{~A}-\mathrm{H} 30 \mathrm{D}$ & 109.5 \\
\hline $\mathrm{C} 29 \mathrm{~A}-\mathrm{C} 30 \mathrm{~A}-\mathrm{H} 30 \mathrm{E}$ & 109.5 \\
\hline $\mathrm{H} 30 \mathrm{D}-\mathrm{C} 30 \mathrm{~A}-\mathrm{H} 30 \mathrm{E}$ & 109.5 \\
\hline $\mathrm{C} 29 \mathrm{~A}-\mathrm{C} 30 \mathrm{~A}-\mathrm{H} 30 \mathrm{~F}$ & 109.5 \\
\hline $\mathrm{H} 30 \mathrm{D}-\mathrm{C} 30 \mathrm{~A}-\mathrm{H} 30 \mathrm{~F}$ & 109.5 \\
\hline $\mathrm{H} 30 \mathrm{E}-\mathrm{C} 30 \mathrm{~A}-\mathrm{H} 30 \mathrm{~F}$ & 109.5 \\
\hline
\end{tabular}




\begin{tabular}{|c|c|c|c|}
\hline $\mathrm{C} 15-\mathrm{N} 2-\mathrm{N} 3-\mathrm{C} 17$ & $-0.9(2)$ & $\mathrm{N} 3-\mathrm{N} 2-\mathrm{C} 15-\mathrm{C} 16$ & $1.2(2)$ \\
\hline $\mathrm{C} 18-\mathrm{N} 2-\mathrm{N} 3-\mathrm{C} 17$ & $-174.05(16)$ & $\mathrm{C} 18-\mathrm{N} 2-\mathrm{C} 15-\mathrm{C} 16$ & $173.39(17)$ \\
\hline $\mathrm{C} 14-\mathrm{N} 1-\mathrm{C} 1-\mathrm{C} 2$ & $-179.41(19)$ & $\mathrm{N} 3-\mathrm{N} 2-\mathrm{C} 15-\mathrm{C} 14$ & $-179.04(15)$ \\
\hline $\mathrm{C} 26-\mathrm{N} 1-\mathrm{C} 1-\mathrm{C} 2$ & $-10.4(3)$ & $\mathrm{C} 18-\mathrm{N} 2-\mathrm{C} 15-\mathrm{C} 14$ & $-6.9(3)$ \\
\hline $\mathrm{C} 14-\mathrm{N} 1-\mathrm{C} 1-\mathrm{C} 6$ & $1.1(2)$ & $\mathrm{C} 7-\mathrm{C} 14-\mathrm{C} 15-\mathrm{N} 2$ & $-82.5(2)$ \\
\hline $\mathrm{C} 26-\mathrm{N} 1-\mathrm{C} 1-\mathrm{C} 6$ & $170.05(17)$ & $\mathrm{N} 1-\mathrm{C} 14-\mathrm{C} 15-\mathrm{N} 2$ & $93.0(2)$ \\
\hline $\mathrm{N} 1-\mathrm{C} 1-\mathrm{C} 2-\mathrm{C} 3$ & $-179.1(2)$ & $\mathrm{C} 7-\mathrm{C} 14-\mathrm{C} 15-\mathrm{C} 16$ & $97.1(3)$ \\
\hline $\mathrm{C} 6-\mathrm{C} 1-\mathrm{C} 2-\mathrm{C} 3$ & $0.4(3)$ & $\mathrm{N} 1-\mathrm{C} 14-\mathrm{C} 15-\mathrm{C} 16$ & $-87.3(2)$ \\
\hline $\mathrm{C} 1-\mathrm{C} 2-\mathrm{C} 3-\mathrm{C} 4$ & $-0.2(3)$ & $\mathrm{N} 2-\mathrm{C} 15-\mathrm{C} 16-\mathrm{C} 17$ & $-1.0(2)$ \\
\hline $\mathrm{C} 2-\mathrm{C} 3-\mathrm{C} 4-\mathrm{C} 5$ & $-0.2(4)$ & $\mathrm{C} 14-\mathrm{C} 15-\mathrm{C} 16-\mathrm{C} 17$ & $179.33(18)$ \\
\hline $\mathrm{C} 3-\mathrm{C} 4-\mathrm{C} 5-\mathrm{C} 6$ & $0.4(3)$ & $\mathrm{N} 2-\mathrm{N} 3-\mathrm{C} 17-\mathrm{C} 16$ & $0.3(2)$ \\
\hline $\mathrm{C} 4-\mathrm{C} 5-\mathrm{C} 6-\mathrm{C} 1$ & $-0.2(3)$ & $\mathrm{C} 15-\mathrm{C} 16-\mathrm{C} 17-\mathrm{N} 3$ & $0.5(2)$ \\
\hline $\mathrm{C} 4-\mathrm{C} 5-\mathrm{C} 6-\mathrm{C} 7$ & $180.0(2)$ & $\mathrm{C} 15-\mathrm{N} 2-\mathrm{C} 18-\mathrm{C} 19$ & $129.10(19)$ \\
\hline $\mathrm{N} 1-\mathrm{C} 1-\mathrm{C} 6-\mathrm{C} 5$ & $179.39(16)$ & $\mathrm{N} 3-\mathrm{N} 2-\mathrm{C} 18-\mathrm{C} 19$ & $-59.1(2)$ \\
\hline $\mathrm{C} 2-\mathrm{C} 1-\mathrm{C} 6-\mathrm{C} 5$ & $-0.2(3)$ & $\mathrm{C} 15-\mathrm{N} 2-\mathrm{C} 18-\mathrm{C} 25$ & $-116.7(2)$ \\
\hline $\mathrm{N} 1-\mathrm{C} 1-\mathrm{C} 6-\mathrm{C} 7$ & $-0.7(2)$ & N3-N2-C18-C25 & $55.1(2)$ \\
\hline $\mathrm{C} 2-\mathrm{C} 1-\mathrm{C} 6-\mathrm{C} 7$ & $179.69(17)$ & $\mathrm{N} 2-\mathrm{C} 18-\mathrm{C} 19-\mathrm{C} 20$ & $-61.1(3)$ \\
\hline $\mathrm{C} 5-\mathrm{C} 6-\mathrm{C} 7-\mathrm{C} 14$ & $180.0(2)$ & $\mathrm{C} 25-\mathrm{C} 18-\mathrm{C} 19-\mathrm{C} 20$ & $-178.4(2)$ \\
\hline $\mathrm{C} 1-\mathrm{C} 6-\mathrm{C} 7-\mathrm{C} 14$ & $0.11(19)$ & $\mathrm{N} 2-\mathrm{C} 18-\mathrm{C} 19-\mathrm{C} 24$ & $117.45(18)$ \\
\hline $\mathrm{C} 5-\mathrm{C} 6-\mathrm{C} 7-\mathrm{C} 8$ & $0.8(3)$ & $\mathrm{C} 25-\mathrm{C} 18-\mathrm{C} 19-\mathrm{C} 24$ & $0.1(2)$ \\
\hline $\mathrm{C} 1-\mathrm{C} 6-\mathrm{C} 7-\mathrm{C} 8$ & $-179.11(17)$ & $\mathrm{C} 24-\mathrm{C} 19-\mathrm{C} 20-\mathrm{C} 21$ & $-1.3(3)$ \\
\hline $\mathrm{C} 14-\mathrm{C} 7-\mathrm{C} 8-\mathrm{C} 13$ & $-40.8(3)$ & $\mathrm{C} 18-\mathrm{C} 19-\mathrm{C} 20-\mathrm{C} 21$ & $177.1(2)$ \\
\hline $\mathrm{C} 6-\mathrm{C} 7-\mathrm{C} 8-\mathrm{C} 13$ & $138.22(19)$ & $\mathrm{C} 19-\mathrm{C} 20-\mathrm{C} 21-\mathrm{C} 22$ & $0.8(4)$ \\
\hline $\mathrm{C} 14-\mathrm{C} 7-\mathrm{C} 8-\mathrm{C} 9$ & $140.51(19)$ & $\mathrm{C} 20-\mathrm{C} 21-\mathrm{C} 22-\mathrm{C} 23$ & $0.2(4)$ \\
\hline $\mathrm{C} 6-\mathrm{C} 7-\mathrm{C} 8-\mathrm{C} 9$ & $-40.4(3)$ & $\mathrm{C} 21-\mathrm{C} 22-\mathrm{C} 23-\mathrm{C} 24$ & $-0.8(4)$ \\
\hline $\mathrm{C} 13-\mathrm{C} 8-\mathrm{C} 9-\mathrm{C} 10$ & $0.0(3)$ & $\mathrm{C} 22-\mathrm{C} 23-\mathrm{C} 24-\mathrm{C} 19$ & $0.3(3)$ \\
\hline $\mathrm{C} 7-\mathrm{C} 8-\mathrm{C} 9-\mathrm{C} 10$ & $178.73(18)$ & $\mathrm{C} 22-\mathrm{C} 23-\mathrm{C} 24-\mathrm{N} 4$ & $-179.4(2)$ \\
\hline $\mathrm{C} 8-\mathrm{C} 9-\mathrm{C} 10-\mathrm{C} 11$ & $1.6(3)$ & $\mathrm{C} 20-\mathrm{C} 19-\mathrm{C} 24-\mathrm{C} 23$ & $0.8(3)$ \\
\hline $\mathrm{C} 9-\mathrm{C} 10-\mathrm{C} 11-\mathrm{C} 12$ & $-1.7(3)$ & $\mathrm{C} 18-\mathrm{C} 19-\mathrm{C} 24-\mathrm{C} 23$ & $-177.95(19)$ \\
\hline $\mathrm{C} 9-\mathrm{C} 10-\mathrm{C} 11-\mathrm{F} 1$ & $179.68(19)$ & $\mathrm{C} 20-\mathrm{C} 19-\mathrm{C} 24-\mathrm{N} 4$ & $-179.49(18)$ \\
\hline $\mathrm{F} 1-\mathrm{C} 11-\mathrm{C} 12-\mathrm{C} 13$ & $178.76(18)$ & $\mathrm{C} 18-\mathrm{C} 19-\mathrm{C} 24-\mathrm{N} 4$ & $1.8(2)$ \\
\hline $\mathrm{C} 10-\mathrm{C} 11-\mathrm{C} 12-\mathrm{C} 13$ & $0.1(3)$ & $\mathrm{C} 25-\mathrm{N} 4-\mathrm{C} 24-\mathrm{C} 23$ & $176.4(2)$ \\
\hline $\mathrm{C} 11-\mathrm{C} 12-\mathrm{C} 13-\mathrm{C} 8$ & $1.6(3)$ & $\mathrm{C} 25-\mathrm{N} 4-\mathrm{C} 24-\mathrm{C} 19$ & $-3.3(2)$ \\
\hline $\mathrm{C} 9-\mathrm{C} 8-\mathrm{C} 13-\mathrm{C} 12$ & $-1.7(3)$ & $\mathrm{C} 24-\mathrm{N} 4-\mathrm{C} 25-\mathrm{O} 1$ & $-175.0(2)$ \\
\hline $\mathrm{C} 7-\mathrm{C} 8-\mathrm{C} 13-\mathrm{C} 12$ & $179.65(17)$ & $\mathrm{C} 24-\mathrm{N} 4-\mathrm{C} 25-\mathrm{C} 18$ & $3.3(2)$ \\
\hline $\mathrm{C} 6-\mathrm{C} 7-\mathrm{C} 14-\mathrm{N} 1$ & $0.55(19)$ & $\mathrm{N} 2-\mathrm{C} 18-\mathrm{C} 25-\mathrm{O} 1$ & $54.6(3)$ \\
\hline $\mathrm{C} 8-\mathrm{C} 7-\mathrm{C} 14-\mathrm{N} 1$ & $179.78(16)$ & $\mathrm{C} 19-\mathrm{C} 18-\mathrm{C} 25-\mathrm{O} 1$ & $176.3(2)$ \\
\hline $\mathrm{C} 6-\mathrm{C} 7-\mathrm{C} 14-\mathrm{C} 15$ & $176.46(17)$ & $\mathrm{N} 2-\mathrm{C} 18-\mathrm{C} 25-\mathrm{N} 4$ & $-123.82(18)$ \\
\hline $\mathrm{C} 8-\mathrm{C} 7-\mathrm{C} 14-\mathrm{C} 15$ & $-4.3(3)$ & $\mathrm{C} 19-\mathrm{C} 18-\mathrm{C} 25-\mathrm{N} 4$ & $-2.0(2)$ \\
\hline $\mathrm{C} 1-\mathrm{N} 1-\mathrm{C} 14-\mathrm{C} 7$ & $-1.0(2)$ & $\mathrm{C} 1-\mathrm{N} 1-\mathrm{C} 26-\mathrm{C} 27$ & $62.8(3)$ \\
\hline $\mathrm{C} 26-\mathrm{N} 1-\mathrm{C} 14-\mathrm{C} 7$ & $-170.54(16)$ & $\mathrm{C} 14-\mathrm{N} 1-\mathrm{C} 26-\mathrm{C} 27$ & $-129.9(2)$ \\
\hline $\mathrm{C} 1-\mathrm{N} 1-\mathrm{C} 14-\mathrm{C} 15$ & $-177.30(15)$ & $\mathrm{C} 1-\mathrm{N} 1-\mathrm{C} 26-\mathrm{C} 28$ & $-65.4(3)$ \\
\hline $\mathrm{C} 26-\mathrm{N} 1-\mathrm{C} 14-\mathrm{C} 15$ & $13.2(3)$ & $\mathrm{C} 14-\mathrm{N} 1-\mathrm{C} 26-\mathrm{C} 28$ & $102.0(2)$ \\
\hline
\end{tabular}


Hydrogen-bond geometry $\left(\AA,{ }^{\circ}\right)$

\begin{tabular}{lllll}
\hline$D-\mathrm{H} \cdots A$ & $D-\mathrm{H}$ & $\mathrm{H} \cdots A$ & $D \cdots A$ & $D-\mathrm{H} \cdots A$ \\
\hline $\mathrm{N} 4-\mathrm{H} 1 N 1 \cdots \mathrm{O} 2^{\mathrm{i}}$ & $0.85(2)$ & $1.92(3)$ & $2.750(19)$ & $165(2)$ \\
$\mathrm{O} 2-\mathrm{H} 1 O 2 \cdots \mathrm{O} 1^{\mathrm{ii}}$ & $0.98(9)$ & $1.67(9)$ & $2.650(2)$ & $172(11)$ \\
\hline
\end{tabular}

Symmetry codes: (i) $-x+1,-y+1,-z+1$; (ii) $x, y-1, z$. 